\title{
L'enseignement des structures préfabriquées en français langue étrangère à travers une approche actionnelle
}

\section{The teaching of prefabricated structures in French as a foreign language through an action-based approach}

\author{
Antonio García Fernández \\ Universidad Nacional de Educación a Distancia (Madrid) \\ agarcia@flog.uned.es
}

\begin{abstract}
This study is about the current teaching of prefabricated structures in French Foreign Language through an action-oriented approach based on the use of applications such as $i M o$ vie, Touchcast and other creative audiovisual applications. This study will specify, first, the current situation of phraseology in the learning of a foreign language; second, the importance of ready-made expressions in social interaction; then, the link between lexicon and culture in phraseological expressions; and finally, the implementation of the teaching of these expressions through an action-centered perspective.
\end{abstract}

\section{Key words}

phraseology, prefabricated structures, actionoriented approach, video, ICT.

\begin{abstract}
Resumen
El presente estudio nos habla sobre la enseñanza actual de las estructuras prefabricadas en Francés Lengua Extranjera (FLE) a través de un enfoque orientado a la acción basado en el uso de aplicaciones como iMovie, Touchcast y otras aplicaciones de creación audiovisual. Este estudio precisará, primeramente, la situación actual de la fraseología en el aprendizaje de una lengua extranjera; a continuación, la importancia de las expresiones prefabricadas en la interacción social; seguidamente, la relación entre léxico y cultura en las expresiones fraseológicas; y finalmente, la puesta en marcha de la enseñanza de estas expresiones a través de una perspectiva centrada en la acción.

Palabras clave

fraseología, structures préfabriquées, approche actionnelle, video, TIC.
\end{abstract}




\section{Introduction}

Depuis le début des approches communicatives dans les dernières décennies du $\mathrm{XX}^{\mathrm{e}}$ siècle, l'acquisition des compétences communicatives est considérée comme l'objectif essentiel de l'enseignement et de l'apprentissage des langues. Il est certain que le lexique occupe un élément central dans nos classes de langues étrangères. À cet égard, il faut souligner que "la place de la phraséologie dans le système langagier, et tout particulièrement sa relation au lexique, est une question importante pour la linguistique en général, mais aussi pour la didactique de la langue" (Lewis, 1997; Granger, 2011 cité par Polguère, 2016: 7). Grâce à l'intérêt croissant pour l'enseignement des expressions phraséologiques, la recherche centrée sur cette composante s'est multipliée et elle a apporté une série de facteurs à prendre en compte pour poursuivre un enseignement et un apprentissage efficaces.

À l'heure actuelle, l'enseignement du lexique envisage la profondeur de la connaissance et la richesse lexicale. Cette richesse concerne tous les aspects qui implique la connaissance d'une unité lexicale: prononciation, structure morphologique, signification, combinatoire, valeur discursive et culturelle, etc. Tous ces aspects doivent se tenir en compte et s'intégrer dans les différentes tâches afin d'apprendre, stocker et fixer les expressions à travers les significations et sa combinatoire avec d'autres mots.

L'enseignement du lexique ne se limite plus à montrer uniquement les formes et les significations des mots comme auparavant, mais cet enseignement commence de plus en plus à faire attention à leur combinatoire et particulièrement à ce que l'on appelle les collocations, locutions ou d'autres expressions phraséologiques.

Les deux types d'expressions phraséologiques les plus connues et étudiées jusqu'à présent sont les locutions et les collocations. Cependant, le lexique comprend aussi certaines structures qui acquièrent aussi une valeur totalement pragmatique, c'est-à-dire qu'elles sont associées à un contexte communicatif spécifique: les clichés linguistiques.

Les clichés linguistiques jouent donc un rôle essentiel dans l'activité linguistique, en tant que pratique sociale. Parce que c'est au niveau même du contenu conceptuel du message que se produit le figement et parce que le cliché est un énoncé en soi, son emploi relève véritablement de la pratique rituelle alors que l'emploi des autres phrasèmes correspond plutôt à l'observance simple des règles du jeu linguistique. (Polguère, 2016: 9)

D'ailleurs, nous savons que près de la moitié de la production linguistique quotidienne est constituée de formules fixes. Cette production est formée par des structures de mots préfabriqués qui sont récupérées de la mémoire en bloc (Wray, 2002: 9), par exemple: un instant, je vous en prie, je vous remercie, etc. Ainsi, nous connaissons déjà des notions telles que séquences préfabriquées, formule toute faite, speech formula ou différents types d'unités lexicales: collocations, locutions ou autres expressions idiomatiques (Polguère, 2016: 11). 
Par conséquent, dans ce travail nous nous concentrerons généralement sur ces expressions de nature pragmatique.

En outre, deux aspects vont marquer notre étude: l'approche actionnelle et l'usage des technologies de l'information et de la communication (TIC). D'un côté, l'approche actionnelle acquiert aussi une fonction incontournable dans l'enseignement de langues vivantes. Cette perspective va permettre une nouvelle vision à travers l'acquisition de diverses compétences, non seulement de compétences générales, mais aussi une compétence communicative, linguistique, sociolinguistique et pragmatique. D’un autre côté, la société dans laquelle nous vivons a subi de nombreux changements, en particulier dans la société de l'information, caractérisée par les TIC. Cependant, ces dernières années, la présence de ces TIC dans le processus d'apprentissage-apprentissage est progressivement accrue. Cette étude visera aussi à démontrer l'application des TIC, en tant que moyen, afin de promouvoir un apprentissage plus individualisé, de fournir une interaction sociale entre les apprenants, et de montrer une série d'outils qui seront utilisés pour le développement du processus d'enseignement-apprentissage, dans ce cas, des expressions phraséologiques.

\section{Les structures préfabriquées dans des situations de communication}

Dans des situations de communication, il est normal de constater tout type de phrasèmes. Dans le cadre de la Théorie Sens-Texte' ${ }^{1}$ (Mel'čuk, 1997), les phrasèmes décrivent les combinaisons non libres de la langue:

Un phrasème est une expression non libre, c'est-à-dire une combinaison de signes linguistiques où au moins un signe n'est pas sélectionné individuellement, pour son signifié, indépendamment des autres signes avec lesquels il est combiné. (Polguère, 2016: 3)

Premièrement, nous distinguons deux grandes classifications des phrasèmes selon le type de restriction (Mel'čuk, 2013: 3): les phrasèmes lexicaux (contraints par rapport à la signification) et les phrasèmes sémantico-lexicaux (contraints par rapport au message conceptuel), et les phrasèmes sémantiquement compositionnels et les phrasèmes non compositionnels. Les phrasèmes lexicaux sont divisés en même temps en phrasèmes non compositionnels (colocation et locutions) et les phrasèmes sémantico-lexicaux en clichés.

Les phrasèmes lexicaux, au niveau sémantique, sont constitués par les colocations et les locutions, tandis que les phrasèmes au niveau sémantico-lexicaux, au niveau de la représentation conceptuelle, sont formés par les clichés.

Ce deuxième groupe de clichés recueille tous les énoncés à caractère pragmati-

1 La théorie Sens-Texte (Mel'čuk, 1997) consiste à une approche théorie linguistique, “dont un des axiomes est la prise en compte systématique de la perspective du Locuteur (et non du Destinataire, de l'interpréteur) dans l'étude des phénomènes linguistiques" (Polguère, 2015: 2). 
ques, c'est-à-dire ces énoncés sont liés à des situations spécifiques de communication et, ainsi, nommés pragmathèmes. C'est le cas, par exemple, des expressions comme: bonne journée, joyeux anniversaire ou défense de stationner, qui se caractérisent par son emploie dans un contexte donné: une salutation, un anniversaire et une signalétique, respectivement.

D’un autre côté, selon sa particularité de composition, les phrasèmes peuvent être aussi compositionnels ou non-compositionnels. Par exemple, le cliché "peinture fraîche" est un phrasème compositionnel, étant donné que sa signification est le résultat de la signification de chaque composante lexicale.

Dans ce cas, cet énoncé fonctionne comme un pragmathème dû à son emploi dans un contexte d'énonciation donné. Par ailleurs, la non compositionnalité n'implique pas nécessairement l'opacité sémantique ${ }^{2}$ du phasème. Par exemple, la colocation faire une blague, dont le sémantisme est déduit par la lexie blague est complètement transparent.

Dans ce sens, les expressions préfabriquées sont des séquences qui sont stockées et relèvent de la mémoire comme un tout pour son emploi à un moment donné:

\begin{abstract}
A sequence, continuous or discontinuous, of words or other elements, which is, or appears to be, prefabricated: that is, stored and retrieved whole from memory at the time of use, rather than being subject to generation or analysis by the language grammar. (Wray, 2002: 9)
\end{abstract}

Par exemple, dans le suivant exemple, nous pouvons observer un discours développé à la boulangerie. Ce discours est développée dans une situation où nous pouvons repérer des expressions qui conforment une communication ritualisée:

(1a) Situation 1: Chez le boulanger

- Bonjour, madame!

- Bonjour! Je voudrais acheter le gâteau aux pommes que j'ai vu hier.

- Désolé madame, mais il n'y a plus de gâteaux aux pommes.

- Oh non! C'est pas vrai! Ce n'est pas de chance!

- Je vais regarder dedans au cas où. Un moment!

- Ok,j'attends!

- C'est dommage, madame, j'ai vendu le dernier il y a vingt minutes.

- Oh non. Alors, quels gâteaux avez-vous?

- Nous avons ce gâteau au chocolat, au citron et à la crème.

- Ça a l'air si bon!

- Oui, ça plaît beaucoup!

2 Opacité sémantique, appelée aussi non compositionnalité. Ce sont plusieurs les applications possibles sur la transparence ou l'opacité sémantique d'une expression: cela va dépendre du plan dénominatif, du plan du sens descriptifs ou du plan de la subsistance du sens littéral en ce qui concernent les métaphores (Kleiber, 2010: 137). 
Anales de Filología Francesa, n. ${ }^{\circ}$ 29, 2021

ANTONIO GARCÍA FERNÁNDEZ

- Ok, je prends ce gâteau au chocolat!

- C'est pour un anniversaire? Est-ce vous avez besoin de bougies?

- Oui, c'est mon anniversaire!

- Oh! Joyeux anniversaire!

- Merci beaucoup!

- Et avec ceci?

- C'est tout, merci!

- Ok, ce sont $25 €$.

- Voilà!

- Merci beaucoup!

- Je vous en prie. Bonne journée! Profitez bien de ton anniversaire!

- Merci! Bonne journée!

Dans cette situation, au cours du processus d'interaction orale, nous trouvons de nombreuses expressions idiomatiques qui sont purement préfabriquées dans le contenu et aussi dans la forme, telles que:

(2a) Je vous en prie.

(2b) Et avec ceci?

(2c) C'est dommage.

(2d) Un moment.

(2e) Pas de soucis.

(2f) Joyeux anniversaire!

Ces phrasèmes correspondent aux clichés linguistiques. Ils sont des énoncés préfabriqués et sémantiquement compositionnels et idiomatiques. Cependant, les clichés ne sont pas entièrement figés ${ }^{3}$, car ils peuvent admettre de modifications dans sa structure lexicosémantique si l'on ajoute des modificateurs qui peuvent fonctionner comme des énoncés intégrés dans une phrase comme compléments (Polguère, 2016: 5).

Dès lors, ces séquences correspondent à des formes préfabriquées, c'est-à-dire à de vrais clichés linguistiques, car lors d'une conversation, le locuteur ne construit pas l'énoncé

3 "Une séquence est figée du point de vue syntaxique quand elle refuse toutes les possibilités combinatoires ou transformationnelles qui caractérisent habituellement une suite de ce type. Elle est figée sémantiquement quand le sens est opaque ou non compositionnel, c'est-à-dire quand il ne peut pas être déduit du sens des éléments composants" (Gross, 1996: 154). La signification globale de l'énoncé n'est pas le résultat de l'ensemble de significations de chaque élément qui le composent. Cependant, cette fixation n'est pas toujours pleine dans tous les phrasèmes, mais il y a une fixation graduelle, c'est-à-dire une expression peut avoir plus ou moins fixation: “le figement peut être partiel si la contrainte qui pèse sur une séquence donnée n'est pas absolue, s'il existe des degrés de liberté” (Gross, 1996: 154). 
Anales de Filología Francesa, n. ${ }^{\circ}$ 29, 2021

L'ENSEIGNEMENT DES STRUCTURES PRÉFABRIQUÉES EN FRANÇAIS LANGUE ÉTRANGÈRE À TRAVERS...

en question, mais le locuteur le cite directement, car l'ensemble des éléments qui le constitue la communication l'exige (Polguère, 2016: 5).

\section{Les expressions préfabriquées dans l'interaction sociale}

La combinatoire lexicale phraséologique, comme les locutions et les collocations, adopte un rôle important du point de vue sémiotique, mais ce sont surtout les clichés linguistiques ceux qui développent une fonction fondamentale dans l'interaction sociale:

Il est certain que les locutions et les collocations ont un rôle social, puisque le respect ou le non-respect des règles n'est pas neutre dans l'interaction sociale; mais ce sont avant tout les clichés - parce qu'ils sont des contenus préfabriqués avant d'être des segments de langue préfabriqués- qui jouent un rôle sur le plan de l'interaction sociale. (Polguère, 2016: 8)

Ces expressions préfabriquées se caractérisent aussi pour acquérir un caractère pragmatique, c'est-à-dire ils sont employés dans des contextes de communications données. La présence correcte de ces expressions a besoin du respect de conditions séquentielles, contextuelles, sociales, stylistiques et d'autres conditions très spécifiques (Schmale, 2013: 36).

Un cliché linguistique est un énoncé phraséologique sémantiquement compositionnel, mais contraint dans son contenu et sa forme, que le Locuteur doit utiliser pour accomplir un acte illocutoire donné. (Polguère, 2016: 274)

Les phrasèmes les plus récurrents qui partagent ce caractère pragmatique sont ceux qui sont employés dans des actes d'énonciations spécifiques, comme étant: les expressions à valeur prépositionnelle (afin de, au moment où), les proverbes et les lieux communs (Pierre qui roule n'amasse pas mousse), et les expressions à valeur syntagmatique, comme les expressions idiomatiques et les colocations (tourner au tour de pot).

L'emploi d'un cliché correspond à un ensemble de règles dans une interaction sociale. Cet impératif social a été identifié par Wray qui expose:

Humans, being psychologically and socially complex, are unable fully to meet their emotional, mental, and physical needs without involving others. One effective tool for drawing others into behaviors beneficial to us is to employ wordstrings that are in current use in our community. They enable us socially to align ourselves with others (I am like you because I talk like you, so you will want to help me), and as a way of minimizing the risk of misunderstanding, since wordstrings or partly lexicalized frames that have their own semantic entry require less decoding. (2012: 231-231)

Lors du processus de communication, le locuteur a le désir d'exprimer un message ou une émotion, et il décide d'utiliser le langage afin d'atteindre ses objectifs. Ce- 
pendant, si le locuteur ne suit pas les implications de la langue dans un contexte socioculturel donné, il court le risque de paraître un étranger, quelqu'un qui n'appartient pas à ce moment-là à une communauté linguistique qui emploie un type de langage donné (Polguère, 2016: 9).

Il suffit de penser aux actes minimaux de communication qui ont lieu dans une boulangerie, entre amis, au supermarché ou au restaurant pour constater l'importance que ces expressions phraséologiques acquièrent dans ces situations.

Le locuteur doit ainsi avoir une excellente maîtrise de la langue pour pouvoir utiliser ces structures préfabriquées afin d'éviter une marginalisation sociale (Polguère, 2016: 9). En fait, les locuteurs emploient des formules spécifiques par inertie sans s'en rendre compte. C'est le cas des expressions, telles que: d'accord, bonne journée, comment ça va?, c'est pas vrai.

Dans ce sens, Wray (2002: 9) défend que près de la moitié de la production linguistique quotidienne soit préfabriquée. Elle est constituée par de chaînes de mots qui sont récupérées de la mémoire comme un bloc:

Sequence, continuous or discontinuous, of words or other elements, which is, or appears to be, prefabricated: that is, stored and retrieved whole from memory at the time of use, rather than being subject to generation or analysis by the language grammar. (Wray, 2002: 9)

Néanmoins, si à un moment donné l'interlocuteur ne sait pas quoi dire ou il ne sait pas continuer avec le fil de la conversation, il ne gère pas le même rituel linguistique que son interlocuteur. Par conséquent, nous sommes socialement bloqués. Cela se produit également généralement dans certains contextes, lors d'une célébration religieuse ou lors d'une réunion professionnelle. Nous retrouverons des termes typiques du domaine, mais aussi des clichés linguistiques (Polguère, 2016: 9). La maîtrise de ces expressions préfabriquées est donc la clé de l'intégration socioculturelle dans un groupe de locuteurs:

Sans cliché, on se prive de la connivence que procure le fait d'employer les messages préfabriqués qui mettent de la ritualisation dans notre élocution. Et c'est justement la nature de rituel du cliché linguistique qui en détermine véritablement l'importance. (Polguère, 2016: 9)

Nous avons donc affaire à une ritualisation dans les échanges linguistiques qui naissent et évoluent différemment dans chaque société, d'où l'importance de la composante culturelle dans la communication. 


\section{L'importance des expressions préfabriquées à travers l'approche actionnelle}

Dans ce sens, l'acquisition des compétences communicative, linguistique, sociolinguistique et pragmatique vise donc à maîtriser la langue presque comme un locuteur natif. Une maîtrise qui lui permet d'agir efficacement et de manière appropriée dans tout contexte de communication grâce aux structures préfabriquées.

D'ailleurs, c'est aussi l'approche actionnelle prônée par le Cadre Européen Commun de Référence pour les Langues qui estime:

L'utilisateur et l'apprenant d'une langue comme des acteurs sociaux ayant à accomplir des tâches (qui ne sont pas seulement langagières) dans des circonstances et un environnement donné, à l'intérieur d'un domaine d'action particulier. Si les actes de parole se réalisent dans des activités langagières, celles-ci s'inscrivent elles-mêmes à l'intérieur d'actions en contexte social qui seules leur donnent leur pleine signification. Il y a "tâche" dans la mesure où l'action est le fait d'un (ou de plusieurs) sujet(s) qui y mobilise(nt) stratégiquement les compétences dont il(s) dispose(nt) en vue de parvenir à un résultat déterminé. (Conseil de l'Europe, 2000: 10)

Cette perspective actionnelle va permettre de réaliser des actions communes et collectives en langue étrangère, c'est-à-dire, agir avec l'autre. Ainsi, comme nous verrons ci-dessous, les activités seront donc étroitement liées aux activités sociales: "si les actes de parole se réalisent dans des activités langagières, celles-ci s'inscrivent elles-mêmes à l'intérieur d'actions en contexte social qui seules leur donnent leur pleine signification" (Conseil de l'Europe, 2000: 15).

Le passage de l'approche communicative vers une perspective actionnelle permet de développer sur l'apprenant un ensemble de compétences générales (savoir, savoir-faire, savoir-être et savoir-apprendre), mais surtout d'intégrer les compétences communicative, linguistique, sociolinguistique et pragmatique dont il dispose dans des situations et dans des conditions précises afin de réaliser des activités langagières.

En effet, le fait d'être compétent dans une langue implique aussi se débrouiller efficacement dans des situations qui sont constituées des expressions. Cette perspective actionnelle prend donc ainsi en compte les ressources cognitives, affectives, volitives et l'ensemble des capacités que possède et met en œuvre l'acteur social. À travers la réalisation de tâches spécifiques, les apprenants vont développer les différentes composantes de la compétence de communication, s'entraîner aux activités langagières et découvrir des aspects culturels. 


\section{Lexique et culture}

La pratique sociale lors de l'apprentissage d'une langue se caractérise par son origine et par son évolution de manière différente dans chaque société, d'où l'importance de la composante culturelle.

La relation plus étroite entre lexique et culture peut être démontrée à travers ce que nous avons toujours connu comme expressions idiomatiques. Ces expressions phraséologiques sont valables pour tout et elles acquièrent une variété de manifestations qui attire l'attention des apprenants. Dans beaucoup d'entre elles, ces expressions sont constituées des références socioculturelles, telles que:

(3a) C'est clair comme de l'eau de roche

(3b) Mi-figue mi-raisin

(3c) Tourner autour du pot

(3d) Mine de rien

(3e) Autant pour moi

(3f) De fil en aiguille

(3g) Qu'est que tu en dis?

(3h) Somme toute

(3i) Tant s'en faut

(3j) Il y a belle lurette

Ce type d'expressions provient des références historiques et culturelles, car ces expressions sont créées à travers des combinaisons de mots qui expriment un sens spécifique au-delà d'un sens direct. Le fait de connaître une langue suppose être familiarisé avec des expressions que les locuteurs natifs emploient et comprennent dans la vie quotidienne. Elles sont mises en évidence dans les dialogues (3g), elles aident dans les descriptions avec une plus grande expression (3a), elles sont grammaticalisées comme des particules discursives (3h).

C'est pour cela qu'il ne s'agit donc pas de présenter sa didactique à partir de la casuistique ou d'une liste d'expressions phraséologiques liées par thématiques (liste avec des animaux, avec des couleurs, avec des parties du corps), mais de faire attention à la valeur pragmatique des expressions phraséologiques dans le texte et non à leur aspect formel.

Étant donné son caractère pragmatique, ces expressions ne fonctionnent pas isolément, elles sont utilisées dans des textes qui se produisent dans un contexte social et culturel particulier, car le fait de connaître un mot ou une expression implique aussi connaître son emploi. L'accent devrait ainsi être mis sur l'intentionnalité, le registre, l'utilisation, le degré de formalité et l'effet qu'acquièrent ce type d'expressions (Polguère, 2008: 126). Dans ce 
sens, la relation entre lexique et culture est plus profonde: apprendre une langue implique s'approprier d'un nouveau modèle culturel.

À cet égard, c'est l'approche actionnelle qui occupe une place fondamentale. Grâce à l'apprentissage vers l'action et à l'accent mis sur l'agir communicationnel, l'apprenant sera capable de participer à des activités collectives pour accomplir un but partagé, de connaître l'importance donnée à la construction du sens, et, en particulier, d'acquérir des aspects socioculturels et pragmatiques de la communication.

Le CECRL, de son côté, constitue le premier document de référence qui supprime réellement les quatre compétences et développe la langue comme activité collaborative. De cette façon, le CECRL distingue quatre modes de communication: la réception, l'interaction, la production et la médiation. Autrement dit, le CECRL s'éloigne de l'approche traditionnelle de l'enseignement-apprentissage des langues pour adopter une approche fondée sur la co-construction du sens, c'est-à-dire, vers l'action.

En effet, dans ce cas, la médiation est définie comme l'activité du langage consistant à reformuler, oralement ou par écrit, un texte dans le but de faciliter la compréhension mutuelle d'autrui. Sa propre expression linguistique ne le concerne pas, mais son rôle consiste à créer l'espace et les conditions appropriées qui permettent la communication ou l'apprentissage, en collaborant avec ses interlocuteurs pour construire des significations.

Le rôle du locuteur en tant que médiateur consiste donc à encourager les autres à construire du sens, à transmettre des informations, à expliquer des choses aux autres lorsqu'ils ne les comprennent pas, à simplifier quelque chose de trop dense, à résumer quelque chose de trop long et à adapter leur langage dans la foulée. La médiation peut être sociale, culturelle, pédagogique, linguistique ou professionnelle.

\section{Mise en situation}

Sur le plan didactique, dans un cours général de FLE, nous exploitons habituellement un document de base écrit ou oral du point de vue grammatical et lexical, mais aussi phonétique, graphique ou culturel. De cette façon, il est préférable de recourir à la transcription des textes oraux, dans lesquels nous repérons principalement des exemples d'expressions institutionnalisées dans leur contexte pragmatique et discursif. Cependant, comment pouvons-nous aussi enseigner des structures préfabriquées à travers une approche actionnelle?

(4) Situation $2^{4}$ : accepter ou refuser une invitation

- Eh! Regarde! C'est Thomas!

- Ah oui!

- Est-ce qu'on peut l'inviter à ta fête?

- Je ne suis pas sure. Thomas, je ne l'aime pas vraiment.

4 Exemple extrait du manuel de Français Langue Étrangère Parachute 2, Ed. Santillana. 
- $\quad$ Mais non! Il est trop sympa! Tu dois faire un essai!

- Ok! D'accord! J'y vais!

- Thomas, Thomas!

- Ah, Jeanne, Margot, salut! Ça va?

- Oui, ça va! Tu vois, on colle des affiches pour la fête des voisins de notre quartier!

- Une fête?

- Oui! Ça sera une méga fête!

- C'est cool, ça!

- $\quad$ Si tu es libre, tu peux venir! On t'invite!

- Ah merci, c'est sympa!

- Tiens, je te donne une invitation!

- Mais au fait... c'est quel jour exactement?

- Samedi prochain, au parc des Platanes.

- Samedi prochain... le 24?

- Oui, on va faire un pique-nique géant!

- Le matin ou le soir?

- Le soit, bien sûr!

- Oh! C'est pas vrai! Ça, c'est pas de chance! Je regrette, mais ça ne va pas être possible...

- Tu ne peux pas venir? Pourquoi?

- Mes parents ne sont pas là et je dois m'occuper de ma petite sœur..

- Oh non!

- Je suis vraiment désolé, les filles...

- Oh, dommage...

- Moi, je sais, mais je ne peux pas.

- Surtout que notre voisine du troisième, avec qui on va faire un gâteau pour la fête, c'est... Christelle!

- Christelle? Elle habite dans votre immeuble?

- Oui, c'est Christelle Galantin!

- Bon, ben... pas de problème! J'emmène ma sœur... avec votre permission, bien sûr!

- D'accord!

- Super! On se voit!

- Au revoir!

- A samedi!

À travers ce dialogue, nous pouvons repérer beaucoup d'expressions afin d'apprendre comment inviter quelqu'un ou accepter ou refuser une invitation, par exemple, des expres- 
sions comme: on t'invite; tu peux venir; c'est quel jour exactement, c'est dommage, je suis vraiment désolé, je ne peux pas, je regrette, c'est pas de chance. Mais aussi, nous pouvons constater des expressions qui articulent une conversation entre amis, telles que: Oui, ça va!; Tu vois; C'est cool, ça!; on se voit!; pas de problèmes!; j'y vais!; C'est pas vrai! Dans ce sens, tout comme la grammaire, les expressions préfabriquées seront toujours travaillées en contexte, visant à une tâche précise.

C'est pour cela que, enfin de pouvoir recueillir tous les aspects pragmatiques comme l'intentionnalité, le registre, l'utilisation, le degré de formalité et l'effet qu'acquièrent ce type d'expressions, nous proposons, comme exemple, la reconstruction des situations de communications concrètes à travers l'enregistrement vidéo avec un chroma key ${ }^{5}$.

Un chroma est un tissu vert utilisé pour la technique audiovisuelle et qui consiste à extraire la couleur de l'image du fond (généralement vert) et la remplacer par une image ou une vidéo. Cela va permettre d'enregistrer des vidéos n'importe où. Ce sont beaucoup les outils que nous pouvons trouver Touchcast, GreenScreen, Kinemaster, iMovie, et même Tiktok. Ces types d'applications nous permettent de créer des vidéos et de présentations interactives qui peuvent être réalisées par les apprenants et aussi par les enseignants.

Évidemment, nous pouvons utiliser ces applications avec un effet chroma key sans utiliser un tissu vert derrière. Il suffit d'un fond d'une seule couleur pour obtenir l'effet, comme les plateformes de vidéoconférences utilisées pour rencontrer virtuellement d'autres personnes, où nous pouvons ajouter un fond. Cependant, pour un usage plus professionnel et précis, nous utiliserons les applications mentionnées.

De cette façon, étant donné que nous devrons créer une situation réelle de communication, les activités de classe choisies par l'enseignant seront donc de l'intérêt proche de la vie réelle de l'élève et associée à la tâche en fonction des objectifs et des besoins. Il s'agit de mettre en jeu toute sa personnalité, ses savoirs et savoir-faire dans la construction du sens.

À cet égard, le CECRL et la perspective actionnelle accordent à la notion de tâche une importance particulière, car l'accomplissement de tâches suppose l'aboutissement de toute activité langagière (Conseil de l'Europe, 2001). La tâche correspond à une situation-problème réel et adéquat aux divers rythmes que l'apprenant doit résoudre grâce à la coopération et à l'interaction avec les autres acteurs sociaux, c'est-à-dire, ces camarades. Les apprenants doivent s'adapter à la tâche en vue de la réalisation d'un objectif commun et afin de retrouver son équilibre bouleversé par le problème posé, dans ce cas, celui de refuser ou accepter une invitation.

De cette façon, pour le développement de la tâche, le groupe sera divisé en deux ou

5 Évidemment, la technologie avance et, à l'heure actuelle il existe aussi des outils d'enregistrement vidéo avec un effet chroma key, mais sans utiliser un tissu vert derrière. Il suffit d'un fond d'une seule couleur pour obtenir l'effet. Cependant, pour un usage plus professionnel et précis, nous utiliserons les applications mentionnées. 
trois personnes et chaque groupe devra créer et simuler une situation donnée de communication. Ce sont beaucoup les situations que nous pouvons proposer: au supermarché, au restaurant, un appel téléphone, etc.

Il est important de noter que, par rapport au niveau de langue, nous pouvons adapter la tâche au niveau des apprenants. Au niveau plus bas (A1/A2.1), nous pouvons recourir directement à une mémorisation du dialogue et postérieurement à l'interprétation; au niveau intermédiaire (A2/B1), nous pouvons guider les apprenants à la rédaction du discours; et au niveau plus avancé (B2/C1), les apprenants seront capables de créer un dialogue / discours d'une façon autonome. Dans ce cas, pour la conception de la tâche, nous nous centrerons concrètement dans un niveau intermédiaire (A2/B1).

\begin{tabular}{ll}
\hline Niveau & Phases de la tâche selon le niveau de l'apprenant \\
\hline Débutant: A1 & Mémorisation - Interprétation \\
\hline Intermédiaire: A2 & Création guidée - mémorisation - interprétation \\
\hline Seuil: B1 & Création guidée - mémorisation - interprétation \\
\hline Avancé: B2 & Création totale - mémorisation - interprétation \\
\hline Autonome: C1 & Création totale - mémorisation - interprétation \\
\hline
\end{tabular}

Il ne s'agit que d'une proposition appliquée dans une situation réelle d'apprentissage. La tâche commence par un texte modèle qui servira de base à la production d'un nouveau texte. Les deux textes traiteront du même sujet, mais ils ne doivent pas nécessairement appartenir au même type ou au même genre. Les apprenants pourront présenter le texte par exemple sous forme de nouvelles ou dans une conversation entre amis.

La tâche sera divisée en trois parties:

\section{1. Élaboration sous forme écrite du dialogue}

Premièrement, chaque groupe va élaborer sous forme écrite le dialogue (ou en Google Drive), qui sera corrigé postérieurement par l'enseignant. À travers la rédaction du dialogue, les apprenants feront usage des expressions préfabriquées travaillées tout au long de l'unité. Il sera facile de mettre en évidence que le texte doit contenir par exemple des expressions telles que: je suis vraiment désolé, ça ne va pas être possible ou pas de problème. Ainsi, pour une meilleure compréhension et mémorisation de ces expressions, il est conseillé de ne pas les sortir du contexte.

\section{Applications des expressions dans le discours et mémorisation du dialogue}

Une fois ces expressions identifiées et employées dans le discours, les apprenants les appliquent dans le discours oral afin de les mémoriser et de les stocker dans le cerveau. Le rôle de la mémoire est essentiel pour comprendre le processus d'apprentissage. Même si l'on 
ne peut pas ignorer cela, la psycholinguistique nous révèle que le progrès serait plus productif que si les mots sont présentés en contexte (Tréville \& Duquette, 1996: 23). D'ailleurs, d'après Cavalla (2009: 38), la mémoire franchit trois étapes:

a) Premièrement, l'apprenant observe pour la première fois un mot nouveau ou une expression nouvelle.

b) Ensuite, l'apprenant doute et devient un sujet actif, car il découvre de nouveaux sens du mot qu'il venait de discerner à travers le contexte.

c) Et finalement, l'apprenant maîtrise les sens du mot et ses constructions syntaxiques et l'utilise de façon naturelle.

D'ailleurs, A. Wray et M. Perkins (2000) exposent que jusqu'à environ un 70\% de la production linguistique d'un adulte devient formulaic:

If we take formulaicity to encompass, as some do, also the enormous set of 'simple' lexical collocations, [...], then possibly as much as $70 \%$ of our adult native language may be formulaic [...]. A range of corpus studies [...] have shown that the patterning of words and phrases in ordinary language manifests far less variability than could be predicted on the basis of grammar and lexicon alone, and in fact most natural language, written or spoken, appears to consist largely of collocational 'sets' or 'frameworks'. (Wray \& Perkins, 2000: 2)

Le locuteur possède donc dans son esprit ces types d'expressions et les applique en bloc lors du processus de communication, il les cite, car la situation l'implique. C'est pour cela que, si l'apprenant mémorise et acquiert certaines expressions d'une situation donnée, il sera capable d'agir d'une manière naturelle comme un locuteur natif, sans se sentir un étranger (Polguère, 2016: 9). Ainsi, après avoir mémorisé et employé toutes les expressions dans ses dialogues, les apprenants vont acquérir le sens des expressions et de le maitriser d'une façon naturelle.

Ainsi, l'apprenant va prendre en considération un nouveau cadre d'apprentissage qui intègre la dimension sociale de la langue et les éléments paralinguistiques impliqués dans la communication, dans lequel l'apprenant acquiert un rôle principal dans son propre processus d'apprentissage.

\section{Mise en scène}

Finalement, dans cette dernière étape, nous procéderions à l'enregistrement de chaque situation à travers l'application TouchCast. Il suffit d'installer un fond vert ou d'une couleur unie, appliquer l'option du chroma et commencer à enregistrer la situation de communication.

Après la mémorisation du dialogue, les apprenants agissent comme s'il s'agissait d'une situation réelle de communication. Grâce à l'usage d'une image que nous ajouterons 
comme fond sur l'application, les apprenants seront immergés dans un espace de la vie quotidienne, soit dans la rue, soit à l'intérieur d'un endroit, par exemple.

Il est important d'observer que cette simulation va permettre que l'apprenant se place dans un espace "réel" de communication, ce qui implique l'acquisition d'une compétence communicative linguistique, sociolinguistique et pragmatique. L'action engage l'apprenant dans la réalisation d'activités communicatives, car son rôle devient celui d'acteur et protagoniste de son apprentissage.

L'intention communicative ne garantit pas le succès de l'action, car dans chaque situation, l'apprenant devra expérimenter de nouvelles possibilités, ce qui signifie que ses décisions ne sont ni aléatoires ni imprévisibles, ce sont des décisions qui obéissent à un besoin, en particulier, celui de simuler une situation donnée de communication.

D'ailleurs, comme nous pouvons observer, le fait de pouvoir voyager dans la salle de classe va nous permettre une plus grande implication des apprenants, dû à l'intérêt et à la motivation de l'usage des nouvelles ressources pour apprendre le français sans s'en rendre compte grâce à la technologie. On n'exploite que le potentiel d'utilisation de l'application TouchCast en classe, mais on favorise aussi un apprentissage beaucoup plus collaboratif en utilisant adéquatement les TIC.

Il s'agit en somme de travailler le lexique sélectionné de telle manière que l'apprenant l'emploie naturellement, c'est-à-dire avec la forme, les significations, les combinaisons et les compétences appropriées à l'action communicative.

\section{Conclusion}

Pour conclure, grâce à l'intérêt croissant pour l'enseignement des expressions phraséologiques, la recherche centrée sur cette composante s'est multipliée et a apporté une série de facteurs à prendre en compte pour poursuivre un enseignement et un apprentissage efficaces: comme l'approche actionnelle et l'usage des logiciels et outils technologiques.

L'objectif est de pouvoir utiliser la langue étrangère dans des contextes de communication réels, c'est-à-dire vers l'action. L'apprenant sera donc prêt à s'intégrer et à participer dans une variété de contextes et de situations qui stimulent leur développement et de meilleures opportunités dans les domaines personnels, publics, éducatifs ou académiques et professionnels.

En effet, la présente étude montre qu'il ne suffit pas de se centrer sur les relations sémantiques entre mots, il faut aussi se centrer sur la relation avec le contexte afin de créer le message. Il est nécessaire de remplacer les listes de mots et d'expressions avec leurs traductions correspondantes par des séquences préfabriquées en contexte. Le lexique ne revient pas aux mots, mais à des fragments de langage, des blocs ou des séquences. Il ne faut pas réduire la connaissance d'une unité lexicale à son équivalence dans la langue maternelle, mais les 
apprenants doivent prendre vraiment conscience de la relation de ces expressions, de sa combinatoire et de sa valeur pragmatique dans un contexte donné.

Tout au long de cette étude, nous avons tenté de démontrer la présence d'une série des séquences de mots préfabriqués lors d'un échange linguistique, telles que: je vous en prie, et avec ceci?, c'est dommage, un moment, pas de soucis, joyeux anniversaire! Ces expressions sont des séquences stockées et récupérées de la mémoire dans son ensemble, au lieu d'être générées entièrement chaque fois qu'elles sont produites ou soumises aux règles de la grammaire.

En somme, l'enseignement des expressions phraséologique doit donc être un enseignement inductif à partir des textes culturellement chargés en faisant la part à l'autonomie de l'apprenant, selon ses besoins, ses objectifs et ses propres connaissances antérieures, c'est-àdire dans une perspective actionnelle.

\section{Références bibliographiques}

Cavalla, Cristelle, Crozier, Elsa, Dumarest, Danièle, Richou \& Claude. 2009. Le vocabulaire en classe de langue. Paris, CLÉ International.

Conseil de L'Europe. Conseil de la coopération culturelle. Comité de l'Éducation. Division des langues vivantes. 2001. Cadre européen commun de référence pour les langues: apprendre enseigner évaluer. Paris, Didier.

Gross, Gaston. 1996. Les expressions figées en français. Noms composés et autres locutions. Paris, Ophrys.

KLEIBLER, Georges. 2010. "Proverbes: transparece et opacité" in Meta, nº 1 (55), 136-146.

MeL'CuK, Igor. 2013. “Tout ce que nous voulions savoir sur les phrasèmes mais...” in $\mathrm{Ca}$ hiers de lexicologie, $\mathrm{n}^{\circ} 102,129-149$.

Mel'Cuk,, Igor. 2015. "Clichés, an Understudied Subclass of Phrasemes” in Yearbook of Phraseology, $\mathrm{n}^{\circ}$ 6, 55-86.

Polguère, Alain. 2008. Lexicologie et sémantique lexicale. Montréal, les Presses de l’Université de Montréal.

Polguère, Alain. 2015. "Non-compositionnalité: ce sont toujours les locutions faibles qui trinquent". Verbum XXXVII, n 2, 255-278.

Polguère, Alain. 2016a. "Il y a un traître par minou: le statut lexical des clichés linguistiques" in CORELA, COgniton, REprésentation, LAngage. HS-19: < http://corela.revues. org/4486> [21/05/2021].

Polguère, Alain. 2016b. Lexicologie et sémantique lexicale. Notions fondamentales. Montréal, Les Presses de l’Université de Montréal.

SchmaLE, Günter. 2013. “Qu'est-ce qui est préfabriqué dans la langue? Réflexions au sujet d'une définition élargie de la préformation langagière" in Langages, $\mathrm{n}^{\circ} 189$ (1), 27- 45. 
Anales de Filología Francesa, n. ${ }^{\circ}$ 29, 2021

ANTONIO GARCÍA FERNÁNDEZ

Treville, Marie-Claude \& Duquette, Lise. 1996. Enseigner le vocabulaire en classe de langue. Paris, Hachette.

Wray, Alison. 2002. Formulaic Language and the Lexicon, Cambridge, Cambridge University Press.

Wray, Alison. 2012. "What Do We (Think We) Know About Formulaic Language? An Evaluation of the Current State of Play" in Annual Review of Applied Linguistics, $\mathrm{n}^{\circ}$ 32, 231-254. 
